\title{
Editorial: Natur, Gesellschaft, Materialität: aktuelle Herausforderungen der Politischen Ökologie
}

\author{
Sören Becker ${ }^{1}$ and Antje Otto ${ }^{2}$ \\ ${ }^{1}$ Institutionenwandel und regionale Gemeinschaftsgüter, Leibniz-Institut für raumbezogene Sozialforschung \\ Erkner, Erkner, 15537, Deutschland \\ ${ }^{2}$ Geographie und Naturrisikenforschung, Universität Potsdam, Potsdam, 14476, Deutschland
}

Correspondence to: Sören Becker (soeren.becker@leibniz-irs.de)

Published: 17 October 2016

\begin{abstract}
Kurzfassung. This editorial introduces different theoretical strands in political ecology both in English and German speaking contexts. Comparing Marxist with more relational and "new materialist" approaches, it is argued that the various theoretical approaches chosen induce different ways of how the relationship between society and nature, between material and culture is conceptualized. The dialectical perspective derived from Marxism is thereby contrasted with the more emergent, and relativist understandings of actor network theory and assemblage thinking. Besides, the six single contributions in this Special Issue are introduced and five areas for further research are laid out: (1) the multiplicity of materiality, (2) the opposition between dialectic and relativist thinking, (3) the tension between elements and entire socio-material configurations, (4) materiality in the production of space and (5) issues of materiality and power.
\end{abstract}

\section{Hintergrund und Ziele des Themenhefts}

Ob und wie Materialität erfasst werden soll, ist eine der wichtigsten konzeptionellen Fragen der Sozialgeographie. Nach dem „,cultural turn“ (z.B. Kazig und Weichhart, 2009) fordern Diskussionen um den ,neuen Materialismus“ (Barad, 2007; Dolphijn und van der Tuin, 2012) und das „Parlament der Dinge“" (Latour, 2009) etablierte Sichtweisen und Hierarchien zwischen Gesellschaft und Natur heraus. Dieses Themenheft adressiert Aspekte der Frage nach Materialität aus der Sicht einer geographischen Politischen Ökologie. Diese verfolgt die Agenda einer zusammenhängenden Analyse von natürlichen und gesellschaftlichen Bedingungen, Beziehungen und Veränderungen. Dies fordert geradezu dazu heraus, der Materialität von Biosphäre, Atmosphäre, Geosphäre, und Hydrosphäre eine besondere Bedeutung beizumessen. Und doch hat dieser einfache Ausgangspunkt zu einer großen Vielfalt von konzeptionellen Zugängen geführt.

Dieses Themenheft verfolgt ein theoriegeleitetes Interesse, ohne auf bestimmte Theorietraditionen zu fokussieren. Im Mittelpunkt stehen verschiedene Zugänge dazu, wie die Materialität ländlicher und städtischer Naturen in Bezug auf gesellschaftliche Machtverhältnisse und Aushandlungen gefasst werden kann. Wir verstehen Politische Ökologie (oder Political Ecology) selbst nicht als kohärentes Theoriegebäude, sondern vielmehr als ein Feld, in dem der Zusammenhang zwischen gesellschaftlichen und natürlichen Fragen mit einer Vielzahl von epistemischen, theoretischen und methodischen Zugängen bearbeitet wird (Bauriedl in diesem Heft; Bryant, 2015; Robbins, 2004). Dementsprechend bringen wir in diesem Heft verschiedene (post)marxistische, diskurstheoretische und radikal-relationale Ansätze in die Diskussion. Gleichzeitig knüpft dieses Themenheft an bereits einige Jahre zurückliegende Themenhefte und Überblicksartikel zur Politischen Ökologie in der deutschsprachigen Geographie an (Flitner, 1998; Krings, 1999 und dazugehöriges Themenheft; Krings, 2008; Krings und Müller, 2001). Die einzelnen Beiträge des vorliegenden Hefts gehen auf die Fachsitzung „Soziomaterialität, Identitäten und Konflikte: konzeptionelle und methodische Herausforderungen der Politischen Ökologie" auf dem Deutschen Kongress für Geographie Anfang Oktober 2015 in Berlin zurück. 


\section{Was ist Politische Ökologie?}

Die deutschsprachige Diskussion um Politische Ökologie findet vor allem in den Übergangsbereichen zwischen Politikwissenschaften, Umweltsoziologie, Kulturwissenschaften, Gender Studies, Anthropologie und Geographie statt. Innerhalb der deutschsprachigen Geographie wurden Gedanken der Politischen Ökologie in den 1990ern - „mit geraumer Verzögerung“ (Krings, 1998:129) im Vergleich zur angelsächsischen Forschung - eingeführt. Hier waren insbesondere Arbeiten von Geist (z.B. 1992) und Krings (z.B. 1996) wegbereitend. Der Forschungsfokus richtete sich zunächst darauf, die Politische Ökologie für die geographische Entwicklungsländerforschung und weniger präsent für die Wirtschaftsgeographie fruchtbar zu machen sowie in empirischen Studien anzuwenden (z.B. Krings, 1998, 1999; Coy und Krings, 2000; Oßenbrügge, 2007; Ammering et al., 2008). Dieser Forschungsfokus bildet bis heute einen zentralen Schwerpunkt politisch-ökologischer Arbeiten. Daneben wurden politisch-ökologische Studien im Zusammenhang mit der Verwundbarkeitsforschung u.a. im Hinblick auf einzelne Bevölkerungsgruppen durchgeführt (z.B. Bohle und Fünfgeld, 2007) sowie Überschneidungen und Unterschiede der Politischen Ökologie zur Politischen Geographie und geographischen Konfliktforschung herausgearbeitet (Flitner, 2001; Reuber, 2012). Seit einigen Jahren weitet sich das Anwendungsfeld, was sich in Beispielen wie Chillas (2005) und Winters (2015) Analyse von „Stadt-Naturen“, Diskussionen des Nachhaltigkeitsbegriffs aus diskurstheoretischer und feministischer Perspektive (Bauriedl, 2007) sowie das Hinterfragen von Begriffen in der Klimadebatte (s. Beiträge in Bauriedl, 2015) zeigt.

In ihrer Anwendung beschränkt sich die politischökologische Forschung somit längst nicht mehr nur auf ländliche Räume des Globalen Südens, sondern nimmt ebenso Städte und den Globalen Norden in den Blick (Heynen et al., 2006; Heynen, 2014; Zimmer, 2010). Auch methodisch gehen politisch-ökologische Forschungen über eine ,akteursorientierte Mehrebenenanalyse, die in einer Argumentationskette von der internationalen bis zur lokalen Ebene die Handlungslogiken unterschiedlichster Umweltakteure verfolgt“" (Krings, 2000:57) vielfältig hinaus (Zimmer, 2010). Die konzeptionellen Zugänge der Politischen Ökologie haben sich ebenso ausdifferenziert und umfassen neben (neo- und post-)marxistischen Ansätzen (Blaikie und Brookfield, 1987; Harvey, 1996) u.a. handlungstheoretische (Bryant und Bailey, 1997; Dietz und Engels, 2014), diskurstheoretische (Escobar, 1996), feministische (Rocheleau und Edmunds, 1997), neo-materialistische (Bennett, 2010; Mattissek und Wiertz, 2014) und postkoloniale (Lawhon et al., 2014). Daneben ist in der deutschen Diskussion das Konzept der gesellschaftlichen Naturverhältnisse (Görg, 1999, 2008; Wissen, 2011) zentral, das stark auf Gedanken aus der Frankfurter Schule der Kritischen Theorie und Gramscianische Ideen rekurriert.
Trotz der theoretischen, methodischen und empirischen Vielfalt lässt sich ein gemeinsamer Kern in den Arbeiten der Politischen Ökologie und Political Ecology definieren, der zwei Aspekte umfasst. Erstens wird in politischökologischen Studien eine konsequente Verknüpfung von ökologischen Fragestellungen mit gesellschaftlichen Verhältnissen angestrebt. Umweltbezogene Problemstellungen im Zusammenhang mit dem Zugang zu und der Nutzung von natürlichen Ressourcen sowie deren Zerstörung werden dabei u.a. mit politischen und historischen Konstellationen, wie bestehenden gesellschaftlichen Ungleichheiten, politökonomischen Abhängigkeiten und vielfältig ausgeprägten Machtgefällen verknüpft. Zweitens hebt sich die politischökologische Forschung von anderen umweltbezogenen Forschungssträngen durch ihre explizite Normativität ab. Diese leitet sich aus der Kritik bestehender Verhältnisse her und erhält in der Forschungstätigkeit selbst Einfluss (Forsyth, 2008; Krings, 2008). Die Politische Ökologie ist in diesem Sinne keine positiv-neutrale Wissenschaft, sondern selbst durch das ,egalitäre Potential“ (Heynen, 2014:599) motiviert, bestehende Ungleichheiten zu verstehen und zu überwinden.

Seit ihren Anfängen entwickelte sich die Politische Ökologie zu einem Feld, in dem neben dem Fokus auf empirische Arbeiten auch theorieorientierte Diskussionen geführt werden. Diese sind für die Sozialgeographie und für weitere raumbezogene Sozialwissenschaften von Bedeutung. $\mathrm{Zu}$ den wohl bedeutendsten Diskussionspunkten, deren Bearbeitung in der politisch-ökologischen Forschung für die Geographie insgesamt informativ sein kann, zählen die Fragen nach dem Verhältnis zwischen Gesellschaft und Natur sowie die der Einbindung von Materialitäten wie gebauter oder gewachsener Umwelt in die Forschung. Diese Fragen werden in unterschiedlichen konzeptionellen Zugängen sehr verschieden beantwortet. Die Aussagen zu Mensch-UmweltVerhältnissen in polit-ökonomischen und radikal relationalen Ansätzen werden im Folgenden vorgestellt.

\section{Politische Ökologie und die Frage der Materialität}

In der Politischen Ökologie wurde die Verbindung zwischen Gesellschaft und Natur zu Beginn häufig dialektisch gedacht, d.h. im Sinne zweier eigenständiger, aber sich gegenseitig beeinflussender Sphären. So hat die englischsprachige Political Ecology ihre Wurzeln in einer Verschmelzung ökologischer Forschung mit marxistischer Gesellschaftsanalyse. Entsprechend wird häufig auf die frühe Standortbestimmung von Blaikie und Brookfield verwiesen, die Politische Ökologie als eine Verbindung der ,concerns of ecology with a broadly defined political economy" verstehen (1987:17). Diese Entwicklung deckt sich mit der zunehmenden Thematisierung der ökologischen Frage als „zweitem“ Widerspruch im Kapitalismus neben dem Lohnverhältnis (O’Connor, 1991; Foster, 2000). Die dahinter liegende Auffassung geht von kontinuierlichen Inwertsetzungen natürli- 
cher Ressourcen durch kapitalistische Produktion aus (Wissen, 2011). Der Blick wird damit auf eine systematische Verschränkung von ökonomischen und natürlichen Kreisläufen unter ihren jeweiligen spezifischen zeitlichen und räumlichen Bedingungen gelenkt. Häufiges Beispiel war hierbei der $\mathrm{Zu}-$ sammenhang zwischen Umweltzerstörung und Weltmarktintegration (Martinez-Alier, 2002; Harvey, 2003). Analytisch gesehen ist Materialität in dieser Sichtweise jedoch nicht nur Objekt ökonomischer Landnahme, sondern Grundlage aller sozialer Prozesse und Konfigurationen (Swyngedouw, 2006).

Trotzdem entwickelten sich innerhalb der marxistischen Diskussion um die Materialität von Natur verschiedene $\mathrm{Zu}-$ gänge, die jeweils ein unterschiedliches Verständnis über den letztendlichen Stellenwert der Natur ausdrücken (vgl. Wissen, 2008). Während in Neil Smith's (1984) These von der „sozialen Produktion der Natur" Natur vor allem die Rolle eines gestalteten Resultats gesellschaftlicher Prozesse zukam, stellten spätere Arbeiten die Eigenwirkung von natürlichen Elementen und Prozessen sowie die Grenzen gesellschaftlicher Naturaneignung heraus (Castree, 2000; Bakker und Bridge, 2006; Bakker, 2010, s. Schmitt in diesem Band). Vertreter der Urban Political Ecology (Heynen et al., 2006; Swyngedouw, 1996; Swyngedouw and Heynen, 2003) argumentierten hingegen in Richtung einer Auflösung der Übergänge zwischen Natürlichem, Technischem und Sozialem innerhalb der Stadt. Trotz dieser Unterschiede bleibt für diesen Strang der Debatte die Einbettung der betrachteten Prozesse in eine Ungleichheiten produzierende, kapitalistische Wertschöpfung konstitutiv.

Einen anderen Zugang zur Frage der Materialität von Natur entwickelt das vor allem in der deutschen Diskussion rezipierte Konzept der gesellschaftlichen Naturverhältnisse (Görg, 1999, 2008; Wissen, 2011). Ebenfalls auf marxistische Ursprünge zurückgehend, wird die Beziehung zwischen Gesellschaft und Natur hier als ein soziales Verhältnis gefasst, das neben anderen, wie Arbeit, Geschlecht und Staat steht (Brand und Wissen, 2011). Wie dieses Verhältnis bzw. diese Verhältnisse organisiert und institutionalisiert sind, spiegelt gesellschaftliche Machtverhältnisse wider. Der Ansatz der gesellschaftlichen Naturverhältnisse entwickelt daher ein dialektisches Verständnis zwischen natürlichen und sozialen (z.B. institutionellen) Materialitäten (Wissen, 2008). Das Konzept der gesellschaftlichen Naturverhältnisse postuliert die „Nicht-Identität“ von Natur und Gesellschaft (Adorno, 1969). Der Hintergrund dieser Denkfigur ist sowohl ein logischer als auch empirischer. Logisch gesehen müssen zwei Sphären unterschieden werden, wenn zwischen diesen vermittelt, also ein Verhältnis aufgebaut werden soll. So behauptet die Perspektive der gesellschaftlichen Naturverhältnisse „keinesfalls das Ende der Differenz zwischen Natur und Gesellschaft" (Görg, 2008:97). Empirisch gesehen erlangt die Natur ihre Eigenständigkeit aber auch dadurch, dass ihre Materialität nicht beliebig zu modifizieren ist, sondern gegenüber den begrifflichen und technologischen Konstruktionen eine gewisse Widerständigkeit besitzt (Görg,
2008:103; Bakker und Bridge, 2006) und damit auch nicht grenzenlos transformiert und ausgebeutet werden kann (s. Schmitt in diesem Heft).

Waren die verschiedenen Ausformungen der marxistischen Konzeption von Materialität lange Zeit prägend, lenkten andere Perspektiven den Blick stärker auf die diskursive Produktion von Natur(en) (Castree, 2001; Demeritt, 2002; Escobar, 1996). Daneben beanspruchen Ansätze der Akteur-Netzwerk-Theorie (Latour, 2005; Law und Hassard, 1999), der Assemblage-Forschung (Holifield, 2009; McFarlane, 2011) und des „neuen Materialismus“ (Barad, 2007; Dolphijn und van der Tuin, 2012) eine Neudefinition von Materialität. Auch wenn es deutliche Unterschiede zwischen diesen Konzepten gibt (Mattissek und Wiertz, 2014), eint sie eine stark relationale Positionierung. Dem Ruf nach ,morethan-human geographies“ (Braun, 2005) folgend, wird das Primat menschlichen Handelns in Frage gestellt. So werden bisher getrennt gedachte Sektoren oder Bereiche, in soziomaterielle Gefüge (Jöns, 2003; Latour, 2005; Mattissek und Wiertz, 2014; Bertram sowie Münster und Poerting in diesem Heft) aufgelöst und vor allem versucht, den Prozess ihrer Zusammensetzung zu verstehen (DeLanda, 2006; McFarlane, 2011). Nicht allein Menschen oder gesellschaftliche Strukturen wird dabei zugeschrieben, durch Handeln neue Konfigurationen zu erschaffen, sondern Dinge und ,nichtmenschliche" Aktanten können diese Prozesse maßgeblich beeinflussen. Diese Überlegungen führten zu intensiven Debatten u.a. um Materialität, lösten in der Politischen Ökologie aber auch eine provokante Diskussion darüber aus, wie Fragen nach gesellschaftlichen Machtunterschiede in einer rein relationalen Perspektive eingebracht werden können (Holifield, 2009).

Die skizzierten Debatten um marxistische Politische Ökologie, ,neuen“ Materialismus und gesellschaftliche Naturverhältnisse bieten jeweils unterschiedliche Zugänge zu Fragen nach der Definition, den Bedingungen und dem Wirken von natürlichen und sozialen Materialitäten. Diese Fragen werden innerhalb der deutschsprachigen Humangeographie erst seit einigen Jahren unter dem Stichwort ,material turn" intensiver bearbeitet (Kazig und Weichhart, 2009). Doch auch in der deutschsprachigen Politischen Ökologie blieben sie in der Forschungspraxis lange „im Hintergrund, so häufig sie auch theoretisch eingefordert" wurden (Flitner, 2003:225). In der angloamerikanischen Forschung hingegen spielt dieses Themenfeld sowohl in der Politischen Ökologie als auch in der Geographie schon länger eine wichtige Rolle. Markus Wissen konstatierte, dass ,der ,spatial turn“ im deutschen Sprachraum eher konstruktivistisch ausgerichtet" war und ,,die physisch-materielle Raumdimension in Gestalt von Natur und gebauter Umwelt als Materialisierung sozialer Verhältnisse nur wenig thematisiert wurde“" (2008:73). Die Ende der 2000er festgestellte Trendwende (Kazig und Weichhart, 2009; Wissen, 2008) hin zu einem großen Interesse daran, wie Materialitäten und deren Beziehung zur Gesellschaft in sozialwissenschaftliche, humangeographische und 
politisch-ökologische Forschungsarbeiten gefasst und untersucht werden können, wird auf der Grundlage neuerer Theorieentwicklungen in den Einzelbeiträgen dieses Themenhefts aufgegriffen.

\section{Beiträge des Themenhefts und Ausblick}

Die vorliegenden Beiträge fokussieren auf die Vermittlung zwischen dem „Sozialen“ und dem „Natürlichen“ und beleuchten aus unterschiedlichen Perspektiven die Frage der Wirkung bzw. „Agency“ des Materiellen. Dabei werden sowohl aus der anglophonen als auch aus der deutschsprachigen Debatte entlehnte Diskussionen aufgegriffen. Im Gegensatz zu zahlreichen politisch-ökologischen Arbeiten, die stark empirisch ausgerichtet sind, stehen in diesen Beiträgen die konzeptionellen Begriffe und Zugänge und deren methodische Umsetzung im Vordergrund, während empirische Ergebnisse zuvorderst deren Veranschaulichung dienen. Dennoch spiegeln die empirischen Fallstudien gleichzeitig Erweiterungen der Anwendungsfelder innerhalb der Politischen Ökologie wider. So werden vielfältige Regionen und Sektoren abgedeckt: Landwirtschaft und (Wasser)Infrastrukturen im Globalen Süden (z.B. in den Beiträgen von Alina Brad, von Daniel Münster und Julia Poerting sowie von Tobias Schmitt), der postsozialistische Raum im Beitrag von Matthias Schmidt in diesem Heft, aber auch Sperrmüll-Assemblagen in der Bonner Altstadt im Artikel von Erik Bertram in diesem Heft.

$\mathrm{Zu}$ Beginn dieses Themenhefts gibt Sybille Bauriedl einen fundierten Überblick über die Entwicklung der Politischen Ökologie in Deutschland und in der anglophonen Welt. Sie versteht Politische Ökologie als Abgrenzungsbewegung gegenüber sozial- und naturdeterministischen Perspektiven einerseits, aber auch gegenüber ,unpolitischen“ Formen der Mensch-Umwelt-Forschung andererseits. Dem stellt sie eine Konzeption von Politischer Ökologie als herrschaftskritischer Wissenschaft gegenüber - eine Position, die betont, wie gesellschaftliche Herrschaftsverhältnisse in natürliche Phänomene und in die Beziehungen zwischen Menschen und Natur eingewoben sind. Dabei vertieft sie aktuelle Auseinandersetzungen der politischen Ökologie mit Materialität, Macht und Raum.

Diese Diskussion um Materialität und gesellschaftliche Herrschaftsverhältnisse greift auch Tobias Schmitt in seinem Beitrag zu Wasserinfrastrukturen in Nordostbrasilien auf. Er entwickelt die an Foucault angelehnte Dispositivanalyse als Methodik, um verschiedenste Elemente, welche die Entwicklung von Wasserinfrastrukturen beeinflussen, zusammenhängend in den Blick zu nehmen. So verzahnt seine Analyse den Diskurs um Dürre, ungleiche Niederschlagsverhältnisse, institutionelle Regelungen und Handlungen wie das integrierte Wassermanagement, aber ebenso widerständige Praktiken der Aneignung durch marginalisierte gesellschaftliche Gruppen. Er versteht Wasser dabei als ,emblematische Materiali- tät“, als durch Machtverhältnisse konstruiertes Element, dessen Eigenlogik sich jedoch nie gänzlich durch technische Beherrschung und gesellschaftliche Herrschaft einfangen lassen kann. Die Dispositivanalyse wird damit als eine anschlussfähige Methode konzeptualisiert, um die Dialektik zwischen Mensch und Natur, aber auch die Machtförmigkeit gesellschaftlicher Naturverhältnisse genauer zu bestimmen.

Der Beitrag von Alina Brad schließt an diese herrschaftskritischen Perspektiven an und erweitert diese um Fragen der Raumproduktion. Am Beispiel der Palmölproduktion in Indonesien zeigt sie auf, wie gesellschaftliche Machtverhältnisse und Interessen einerseits, und materielle Eigenschaften der Ölpalme wie Anforderungen an Standorte und Möglichkeiten des Transports und der Lagerung andererseits, die räumlichen Strukturen der Palmölproduktion bestimmen. Diese sind wiederum in Prozesse des Rescaling wie die Weltmarktintegration und Reformen im politischen Systems Indonesiens eingebunden. Ihr Beitrag verknüpft dabei der Diskussion um die Produktion von Natur mit Fragen gesellschaftlicher Naturverhältnisse und des State Rescaling. Diese politischen Auseinandersetzungen produzieren eine ungleiche Geographie des Zugangs und der Kontrolle von Gewinnen, Ressourcen und Land.

Die Frage, wie Land als zentraler Untersuchungsgegenstand der Politischen Ökologie und als wichtiger Konfliktgegenstand im Anthropozän konzipiert werden kann, diskutieren Daniel Münster und Julia Poerting in ihrem Beitrag. Auf der Grundlage ihrer Forschungen in Südindien und Nordpakistan beleuchten sie Land aus drei unterschiedlichen Perspektiven: (1) als Ressource in kapitalistischen Prozessen, (2) als Boden, wobei agrarökologische Fragen und alternative, relationale Ontologien der Agrarwirtschaft im Vordergrund stehen und (3) als zusammenhängende Landschaften, die sowohl als Ressource als auch als Gefüge betrachtet werden können. Auf der Grundlage dieser drei Perspektiven, entwickeln Münster und Poerting eine Forschungsagenda für die weitere Betrachtung von Land und Landwirtschaft als Kernbegriffe der Politischen Ökologie. Sie stellen dabei ein relationales Verhältnis zwischen Mensch und Natur heraus und plädieren dafür, agrarwirtschaftliches Land als gleichermaßen diskursiv und materiell zu betrachten.

Eine radikal relationale Perspektive entwickelt Erik Bertram in seinem Beitrag. Er wendet die Methode des nichtrepräsentionalen Denkens (non-representational thinking) auf das Phänomen von Sperrmüll in der Bonner Altstadt an. Auf verschiedenen Streifzügen hat sich der Autor Sperrmüllsammlungen und den sie umgebenden menschlichen Handlungen genähert und begreift den von ihm beobachteten Ausschnitt von Beziehungen zwischen Menschen und Dingen als Gefüge nach Deleuze und Guattari. Der methodisch orientierte Beitrag stellt dabei drei wesentliche Punkte des nichtrepräsentionalen Forschens und deren An- sowie Herausforderungen in den Fokus: das Erschließen, das material thinking sowie das Schreiben und Präsentieren. Bertram zeigt, wie nicht-repräsentionale Ansätze einen neuen, radikal rela- 
tionalen Blick auf das Entstehen und Vergehen vielfältiger Gefüge rund um Sperrmüll und seine Nutzungen frei gibt und mitunter auch (innereuropäische) Wohlstandsunterschiede aufscheinen lässt.

Matthias Schmidt stellt in seinem Beitrag ein neues Anwendungsfeld der Politischen Ökologie dar. Er entwirft das Konzept einer Politischen Ökologie des Postsozialismus - ein Raum, der einerseits weitreichende gesellschaftliche Transformationen sowie Umbrüche und andererseits Persistenzen z.B. hinsichtlich real-sozialistischer Materialitäten, Institutionen oder Naturwahrnehmungen aufweist, die als socialist legacies noch immer prägend wirken. Er stellt die besonderen Bedingungen dieser Region bezogen auf die Gesellschaft-Umwelt-Verhältnisse heraus und entwickelt darauf aufbauend eine Forschungsagenda für die politischökologische Beschäftigung mit Räumen des Postsozialismus. Der Beitrag zeigt zudem auf, dass somit nicht nur postsozialistische Mensch-Umwelt-Verhältnisse besser verstanden und Ansätze für eine gerechtere Entwicklung aufgezeigt werden könnten, sondern die Politische Ökologie auf eigene Schwachstellen wie den Fokus auf kapitalistische Prozesse als hauptsächliche Ursache von Umweltdegradationen oder die Romantisierung lokaler und dörflicher Gemeinschaften hingewiesen werden kann.

Das Themenheft liefert keine definitive Antwort darauf, wie Materialität zu denken sei. Dennoch zeigen die verschiedenen Perspektiven in den einzelnen Aufsätzen einige konzeptionelle Spannungslinien und Desiderate auf. Im Folgenden stellen wir daher in unseren Augen wichtige Ansatzpunkte für die Weiterarbeit an „Materialität“ in der Politischen Ökologie und darüber hinaus dar:

Vielfalt von Materialität: In den Beiträgen des Sonderhefts wird Materialität als weit gefasst verstanden. Entsprechend der Entwicklung in der Politischen Ökologie liefert die Frage, wie sich Natur als Materialität begreifen lassen kann, dabei oft nur einen Ausgangspunkt neben anderen. Zusätzlich werden auch menschlich geschaffene Strukturen, wie gebaute Umwelt, Technologie und Infrastrukturen einbezogen. AuBerdem werden in der marxistischen Tradition auch gesellschaftliche Praxen und Institutionen als ,materiell“ angesehen. Sybille Bauriedls Beitrag in diesem Heft bietet mit seiner Fokussierung auf Herrschaftsverhältnisse einen Ansatz dafür wie dieses Spannungsfeld gefasst werden kann.

Sozio-Materialität als Dialektik oder Gefüge: In diesem Heft werden verschiedene Ansätze dahingehend vertreten, ob Natürliches und Gesellschaftliches als zwei Sphären oder eine Einheit betrachtet werden sollen. Die Spannweite reicht dabei von relationalen Zugängen (z.B. im Beitrag von Erik Bertram zu Sperrmüll-Assemblages) bis hin zu stärker dialektischen Ansätzen (z.B. im Beitrag von Alina Brad). Einen Vermittlungsversuch zwischen diesen Positionen unternimmt Tobias Schmitt, indem er das stärker relationale Konzept der Dispositivanalyse nutzt, um den $\mathrm{Zu}$ sammenhang von gesellschaftlichen Strukturen, Infrastrukturen und Natur genauer zu bestimmen. Wie der Zusam- menhang von gesellschaftlichen und natürlichen Elementen gefasst wird, stellt weiterhin eine Schlüsselfrage der SozioMaterialitätsforschung dar.

Eigenlogik von Elementen vs. Funktionalität der Gesamtheit: Unabhängig von der Gesamtkonzeption unterstreichen alle Autoren dieses Bandes die Eigenlogik oder das eigenständige Wirken einzelner Elemente von soziomateriellen Konfigurationen. Häufig sind dies physische Eigenschaften von natürlichen Gegebenheiten wie die Beschaffenheit von Böden, die Eigenschaften von Wasser oder Ölpalmen. Aber auch sozialen Praxen der Aneignung wird zugeschrieben, unvorhergesehene Ergebnisse zu produzieren, seien es die Landlosen Brasiliens, die für die Landwirtschaft vorgesehenes Wasser aus den Kanälen abzweigen, oder Kulturaktive in Bonn, die aus Sperrmüllelementen ein spontanes Kino im öffentlichen Raum errichten. Wie diese Widerständigkeit nicht nur beschrieben, sondern auch über einzelne Beispiele hinaus erklärt werden kann, bleibt eine große konzeptionelle und methodische Herausforderung nicht nur in der Politischen Ökologie.

Materialität und Raumproduktion: Mit den unterschiedlichen Perspektiven auf Materialität wird auch die Frage nach der Raumproduktion in den Beiträgen sehr verschieden konzeptualisiert. Daniel Münster und Julia Poerting zeigen in ihrem Beitrag anhand des Untersuchungsgegenstandes „Land“ auf, wie divers Raum - analog zur Natur - in der Politischen Ökologie gedacht werden kann. Alina Brad geht davon aus, dass Gesellschaft und Räume jeweils aufeinander formend wirken. Hierbei ist der Aspekt, der in Matthias Schmidts Beitrag deutlich wird, von Interesse, dass Materialitäten aus einer vergangenen (in diesem Fall sozialistischen) Zeit bis heute prägend wirken können und bei der Raumproduktion präsent bleiben. Räumlichen Strukturen wird dabei eine materielle Wirkung zugesprochen.

Materialität und Macht: Die Verortung von Macht vollzieht sich in der Analyse von Materialität je nach gewähltem Ansatz ganz unterschiedlich: Liegt sie in der Wirkungsmacht der einzelnen Elemente von Gefügen, in den globalen ökonomischen Strukturen wie dem Weltmarkt und gesellschaftlichen Herrschaftsverhältnissen, in den Prozessen, die das Zusammenfinden von Gefügen steuern, oder in diskursiven Rahmen von Dispositiven? Letztlich leiten sich aus den unterschiedlichen Zugängen auch unterschiedliche Verhältnisse von Macht ab, eine Gemeinsamkeit scheint lediglich in der Kopplung von Macht und Wirkung zu liegen. Die Konzeption von Macht hat dabei auch einen großen Einfluss darauf, wie der Wandel materieller oder soziomaterieller Strukturen verstanden wird: als eingeschränkte Kontingenz, soziale Auseinandersetzungen um Machtverhältnisse, oder aus der inhärent gegebenen Möglichkeit, die Zusammensetzung von Gefügen zu verändern.

Zusammenfassend plädiert dieses Editorial dafür, Politische Ökologie stärker als bisher auch als Forschungsfeld zu betrachten, das wesentliche Impulse in konzeptionellen Debatten liefern kann. Daneben bleiben empirisch ausgerichtete 
Untersuchungen innerhalb der Politischen Ökologie weiterhin zentral, um Mensch-Umwelt-Verhältnissen in einer politischen Form nachzuvollziehen sowie kritische und alternative Verständnisse und Handlungsangebote aufzuzeigen.

\section{Literatur}

Adorno, T. W.: Stichworte. Kritische Modelle 2. 1. Aufl., Suhrkamp, Frankfurt am Main, Deutschland, 1969.

Ammering, U., Neuburger, M. und Schmitt, T.: Umwelt zwischen Wachstum und Entwicklung. Politische Ökologie von Umweltkonflikten in den Ländern des Globalen Südens, Journal für Entwicklungspolitik, 3, 94-114, 2008.

Bakker, K.: The limits of "neoliberal natures". Debating green neoliberalism, Prog. Hum. Geog., 34, 715-735, 2010.

Bakker, K. und Bridge, G.: Material worlds? Resource geographies and the "matter of nature", Prog. Hum. Geog., 30, 1, 5-27, 2006.

Barad, K.: Meeting the Universe Halfway. Quantum Physics and the Entanglement of Matter and Meaning, Duke University Press, Durham, London, 2007.

Bauriedl, S.: Spielräume nachhaltiger Entwicklung. Die Macht stadtentwicklungspolitischer Diskurse, oekom-Verl., München, Deutschland, 2007.

Bauriedl, S. (Hrsg.): Wörterbuch Klimadebatte, transcript, Bielefeld, Deutschland, 2015.

Bennett, J.: Vibrant matter. A political ecology of things, Duke University Press, Durham, USA, 2010.

Blaikie, P. M. und Brookfield, H. C.: Land degradation and society, Methuen, London, New York, 1987.

Bohle, H.-G. und Fünfgeld, H.: The Political Ecology of Violence: Contested Entitlements and Politicised Livelihoods in Eastern Sri Lanka, Dev. Change, 38, 665-687, 2007.

Brand, U. und Wissen, M.: Die Regulation der ökologischen Krise: Theorie und Empirie der Transformation gesellschaftlicher Naturverhältnisse, in: Österreichische Zeitschrift für Soziologie, 36, 2-34, 2011.

Braun, B.: Environmental issues: writing a more-than-human geography, Prog. Hum. Geog., 29, 635-650, 2005.

Bryant, R. L. (Hrsg.): The International Handbook of Political Ecology, Edward Elgar, Cheltenham, Northampton, 2015.

Bryant, R. L. und Bailey, S.: Third World Political Ecology. An Introduction, Routledge, London, New York, 1997.

Castree, N.: Marxism and the Production of Nature, Capital \& Class, 24, 3, 5-36, 2000.

Castree, N.: Socializing Nature: Theory, Practice, and Politics, in: Social Nature. Theory, Practice, and Politics, Herausgeber: Castree, N. und Braun, B., Blackwell, Malden MA, Oxford, Carlton, 2001.

Chilla, T.: Stadt-Naturen in der Diskursanalyse. Konzeptionelle Hintergründe und empirische Möglichkeiten, Geogr. Z., 93, 183196, 2005.

Coy, M. und Krings, T.: Umweltveränderungen und Politische Ökologie in Entwicklungsländern. Einleitung, in: Lokal verankert - weltweit vernetzt. Tagungsbericht und wissenschaftliche Abhandlung, Herausgeber: Blotevogel, H. und Oßenbrügge, J., Franz Steiner, Stuttgart, Deutschland, 2000.

DeLanda, M.: A new philosophy of society. Assemblage theory and social complexity, Continuum, London, 2006.
Demeritt, D.: What is the ,social construction of nature“? A typology and sympathetic critique, Prog. Hum. Geog., 26, 767-790, 2002.

Dietz, K. und Engels, B.: Immer Ärger wegen der Natur? Für eine gesellschafts- und konflikttheoretische Analyse von Konflikten um Natur, Österreichische Zeitschrift für Politikwissenschaft, 43, 73-90, 2014.

Dolphijn, R. und van der Tuin, I.: New materialism. Interviews et cartographies, 1. ed., Open Humanities Press, Ann Arbor, USA, 2012.

Escobar, A.: Constructing nature. Elements for a poststructural political ecology, in: Liberation ecologies. Environment, development, social movements, Herausgeber: Peet, R. und Watts, W., Routledge, London, New York, 46-68, 1996.

Flitner, M.: Konstruierte Naturen und ihre Erforschung, Geogr. Helv., 53, 89-95, doi:10.5194/gh-53-89-1998, 1998.

Flitner, M.: Politische Geographie und "Political Ecology": Ein Diskussionsbericht, in: Politische Geographie: Handlungsorientierte Ansätze und Critical Geopolitics, Herausgeber: Reuber, P. und Wolkersdorfer, G., Selbstverlag des Geogr. Instituts, Heidelberg, Deutschland, 249-255, 2001.

Flitner, M.: Kulturelle Wende in der Umweltforschung - Aussichten in Humanökologie, Kulturökologie und Politischer Ökologie, in: Kulturgeographie. Aktuelle Ansätze und Entwicklungen, Herausgeber: Gebhardt, H., Reuber, P. und Wolkersdorfer G., Sprektrum Akademischer Verlag, Heidelberg, Berlin, Deutschland, 213-228, 2003.

Forsyth, T.: Political ecology and the epistemology of social justice, Geoforum, 39, 756-764, 2008.

Foster, J. B.: Marx's Ecology. Materialism and Nature, Monthly Press, New York, USA, 2000.

Geist, H.: Die orthodoxe und politisch-ökologische Sichtweise von Umweltdegradierung, Die Erde, 123, 283-295, 1992.

Görg, C.: Gesellschaftliche Naturverhältnisse, 1. Aufl., Westfälisches Dampfboot, Münster, 1999.

Görg, C.: Regulation globaler Naturverhältnisse. Zur Vermittlung von Umwelt und Gesellschaft im globalen Wandel, Berichte zur deutschen Landeskunde 82, 95-113, 2008.

Harvey, D.: Justice, nature, and the geography of difference, Blackwell Publishers, Cambridge, USA, 1996.

Harvey, D.: The new imperialism. Oxford University Press, Oxford, New York, USA, 2003.

Heynen, N.: Urban Political Ecology I. The Urban Century, Prog. Hum. Geog., 38, 598-604, 2014.

Heynen, N., Kaika, M. und Swyngedouw, E. (Hrsg.): In the nature of cities. Urban political ecology and the politics of urban metabolism, Routledge, London, New York, 2006.

Holifield, R.: Actor-Network Theory as a Critical Approach to Environmental Justice: A Case against Synthesis with Urban Political Ecology, Antipode, 41, 637-658, 2009.

Jöns, H.: Mensch-Umwelt-Beziehungen aus einer erweiterten Akteurnetzwerkperspektive, in: Humanökologie. Ansätze zur Überwindung der Natur-Kultur-Dichotomie, Herausgeber: Meusburger, P. und Schwan, T., Franz Steiner Verlag, Wiesbaden, Deutschland, 101-137, 2003.

Kazig, R. und Weichhart, P.: Die Neuthematisierung der materiellen Welt in der Humangeographie, Berichte zur deutschen Landeskunde, 83, 109-128, 2009. 
Krings, T.: Politische Ökologie der Tropenwaldzerstörung in Laos, Petermanns Geographische Mitteilungen, 140, 161-175, 1996.

Krings, T.: Mensch-Umwelt-Beziehungen in den Tropen unter besonderer Berücksichtigung der Politischen Ökologie als Gegenstand der geographischen Entwicklungsforschung, Rundbrief Geographie, 149, 22-25, 1998.

Krings, T.: Ziele und Forschungsfragen der Politischen Ökologie. Editorial zum Themenheft "Politische Ökologie - Neue Perspektiven in der geographischen Umweltforschung", Zeitschrift für Wirtschaftsgeographie, 43, 129-130, 1999.

Krings, T.: Das politisch-ökologische Analysekonzept in der Umweltforschung. Beispiel der städtischen Brennstoffversorgung in Dakar (Senegal), Geographische Rundschau, 52, 56-59, 2000.

Krings, T.: Politische Ökologie. Grundlagen und Arbeitsfelder eines geographischen Ansatzes der Mensch-Umwelt-Forschung, Geographische Rundschau, 60, 4-9, 2008.

Krings, T. und Müller, B.: Politische Ökologie. Theoretische Leitlinien und aktuelle Forschungsfelder, in: Politische Geographie: Handlungsorientierte Ansätze und Critical Geopolitics, Herausgeber: Reuber, P. und Wolkersdorfer, G., Selbstverlag des Geogr. Instituts, Heidelberg, Deutschland, 93-116, 2001.

Latour, B.: Reassembling the social. An introduction to ActorNetwork-Theory, Oxford University Press, Oxford, UK, 2005.

Latour, B.: Das Parlament der Dinge. Für eine politische Theorie, Suhrkamp, Frankfurt, Deutschland, 2009.

Law, J. und Hassard, J.: Actor network theory and after, Blackwell/Sociological Review, Oxford, Malden, 1999.

Lawhon, M., Ernstson, H. und Silver, J.: Provincializing urban political ecology: Towards a situated UPE through African urbanism, Antipode, 46, 497-516, 2014.

Martinez-Alier, J.: The Environmentalism of the Poor. A Study of Ecological Conflicts and Valuation, Edward Elgar, Cheltenham, 2002.

Mattissek, A. and Wiertz, T.: Materialität und Macht im Spiegel der Assemblage-Theorie: Erkundungen am Beispiel der Waldpolitik in Thailand, Geogr. Helv., 69, 157-169, doi:10.5194/gh-69-157$2014,2014$.
McFarlane, C.: The city as assemblage: dwelling and urban space, Environ. Plann. D, 29, 649-671, 2011.

O'Connor, J.: On the two contradictions of capitalism, Capitalism Nature Socialism, 2, 107-109, 1991.

Oßenbrügge, J.: Ressourcenkonflikte ohne Ende? Zur Politischen Ökonomie afrikanischer Gewaltökonomien, Zeitschrift für Wirtschaftsgeographie, 51, 150-162, 2007.

Reuber, P.: Politische Geographie, Ferdinand Schöningh, Paderborn, Deutschland, 2012.

Robbins, P.: Political Ecology. Critical Introduction to Geography, Wiley-Blackwell, Chichester, UK, 2004.

Rocheleau, D. und Edmunds, D.: Women, men and trees: gender, power and property in forest and agrarian landscapes, World Dev., 25, 1351-1371, 1997.

Smith, N.: Uneven development. Nature, capital, and the production of space, Blackwell Publ, Basingstoke, UK, 1984.

Swyngedouw, E.: The city as a hybrid: On nature, society and cyborg urbanization, Capitalism Nature Socialism, 7, 65-80, 1996.

Swyngedouw, E.: Metabolic Urbanization. The Making of Cyborg Cities, in: In the Nature of Cities. Urban political ecology and the politics of urban metabolism, Herausgeber: Heynen, N., Kaika, M. und Swyngedouw, E., Routledge, London, New York, 20-39, 2006.

Swyngedouw, E. und Heynen, N. C.: Urban Political Ecology, Justice and the Politics of Scale, Anitpode, 35, 898-918, 2003.

Winter, K.: Ansichtssache Stadtnatur, transcript, Bielefeld, Deutschland, 2015.

Wissen, M.: Die Materialität von Natur und gebauter Umwelt, in: Kritik und Materialität, Herausgeber: Dimerović, A., Westfälisches Dampfboot, Münster, Deutschland, 2008.

Wissen, M.: Gesellschaftliche Naturverhältnisse in der Internationalisierung des Staates. Konflikte um die Räumlichkeit staatlicher Politik und die Kontrolle natürlicher Ressourcen, Westfälisches Dampfboot, Münster, Deutschland, 2011.

Zimmer, A.: Urban Political Ecology. Theoretical concepts, challenges, and suggested future directions, Erdkunde, 64, 343-354, 2010 . 